\title{
Role of Coping Strategies with Stress in Resiliency and Quality of Life of Female Adolescents with Veteran Father
}

\section{ART ICLE INF O}

\section{Article Type}

Descriptive Study

\section{Authors}

Akbari B.* PhD,

Ghasemi Jobaneh R. ${ }^{1} M S c$, Asadiparvar M. ${ }^{2} M S C$

How to cite this article
Akbari B, Ghasemi Jobaneh R,
Asadiparvar M. Role of Coping
Strategies with Stress in Resilie-
ncy and Quality of Life of Female
Adolescents with Veteran Father.
Iranian Journal of War \& Public
Health.2017;9(4):199-203.

*General Psychology Department, Humanities Faculty, Rasht Branch, Islamic Azad University, Rasht, Iran ${ }^{1}$ Counseling Department, Psychology \& Educational Scineces Faculty, Kharazmi University, Tehran, Iran ${ }^{2}$ General Psychology Department, Humanities Faculty, Rasht Branch, Islamic Azad University, Rasht, Iran

\section{Correspondence}

Address: Psychology Department, Humanities Faculty, Islamic Azad University, Rasht Branch, Darvazaeh Lakan, Rasht, Iran

Phone: +98 (13) 33472099 -

Fax: +98 (13) 33472099

bakbari44@yahoo.com

\section{Article History}

Received: April 26, 2017

Accepted: July 18, 2017

ePublished: November 6, 2017

\section{A B S T R A C T}

Aims Veterans are more susceptible to emotional distress which may affect the well-being of their family members. Coping strategies adopted by adolescents can play an efficient role in their quality of life and psychological state. Therefore, the aim of this study was to investigate the role of coping strategies with stress in psychological resilience and quality of life of female adolescents with veteran father.

Instruments \& Methods This descriptive correlational study was conducted on female students whose father was a veteran in 2015. A population of 170 students was selected using convenience sampling method from Shahed secondary high-schools of Rasht City. Connor-Davidson resilience scale, Endler and Parker coping questionnaire and quality of life questionnaire were used to collect information from the participants. The data were analyzed by SPSS 18 software using Pearson correlation test and multiple regression analysis.

Findings The problem-focused (beta=0.54), emotion-focused (beta=-0.28) and avoidancefocused (beta $=0.14$ ) coping strategies could significantly predict $48 \%$ of variance in resilience. Also, the problem-focused (beta=0.49), emotion-focused (beta=-0.21) and avoidance-focused (beta $=-0.14$ ) coping strategies could significantly predict $30 \%$ of variance in quality of life $(\mathrm{p}<0.01)$.

Conclusion Stress coping strategies predict the psychological resilience and quality of life of female adolescents with veteran father.

Keywords Coping behavior; Resilience, Psychological; Quality of Life; Adolescent

\section{I T A T I O N L I N KS}

[1] The survey of veterans' depression and anxiety and its relationship with veterans' social-economic activities [2] The prevalence of anxiety and depression disorders in the children of veterans of Shiraz, Iran: A case control study [3] Effect of having a post-traumatic stress disordered man on the quality of life, depression, stress, anxiety and structure of the family [4] Assessment and comparison of self esteem and depression in war handicappeds and non war handicappeds children of shahed guidance schools in Sari City [5] Resilience definitions, theory, and challenges: Interdisciplinary perspectives [6] A systematic review of resilience in the physically ill [7] Understanding resilience [8] Relationship between resilience and quality of life in diabetics [9] Health-related quality of life--an introduction [10] Perceived stress, coping, and adjustment in adolescents [11] Relations among wellbeing, avoidant coping, and active coping in a large sample of Australian adolescents [12] Personality and coping [13] Psychological and educational resilience in high vs. low-risk Romanian adolescents [14] Evaluation of Mental health state in veterans family (15-18 Y/O adolescents) [15] Comparison of mental health of high school students according to gender and place of residence [16] Mental health status of high school girl and boy students in boarding schools in Markazi Province [17] Psychometrics properties and normalization of persi version of resiliency questionnaire among athletes and non-athletes students [18] Validation of Endler \& Parker coping scale of stressful situations [19] The short form health survey (SF-36): Translation and validation study of the Iranian version [20] Stressresilience, illness, and coping: A person-focused investigation of young women athletes [21] Resilience, positive coping, and quality of life among women newly diagnosed with gynecological cancers [22] Coping strategies and psychological outcomes: The moderating effects of personal resiliency [23] Coping and resilience of adolescents with congenital heart disease [24] Coping strategies, quality of life and pain in women with breast cancer [25] Relationship between ways of coping and quality of life in married women: Toward mental health promotion [26] Coping styles as correlates of health in high school students 


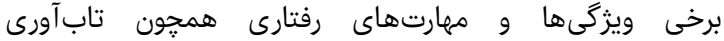

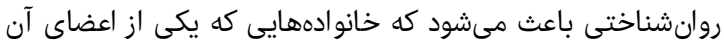

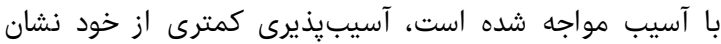

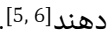

تابآورى بهعنوان توانايى سازگًارى موفقيتآميز با شرايط دشوار

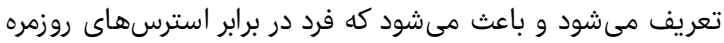

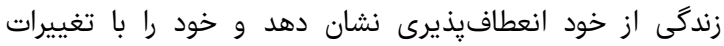

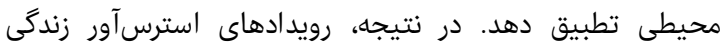

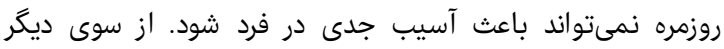

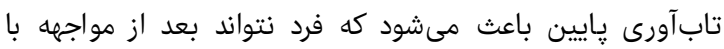

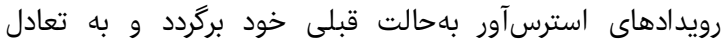

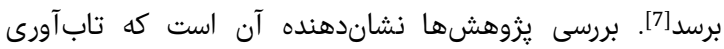

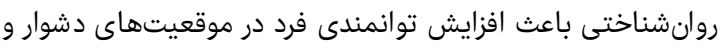

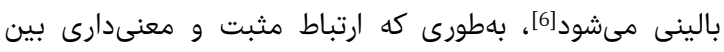

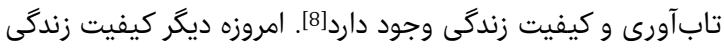

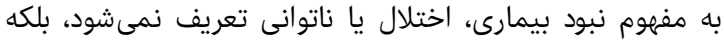

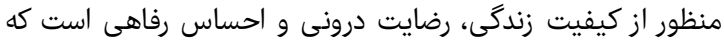

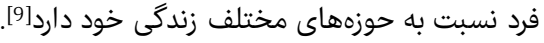

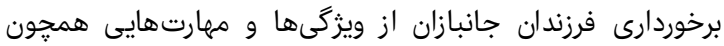

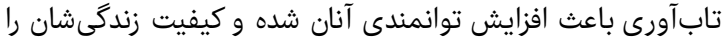

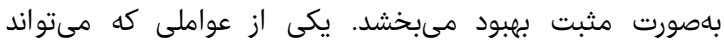

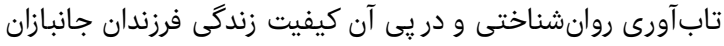

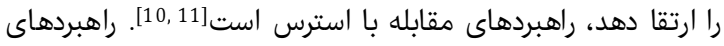

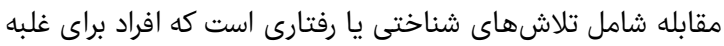

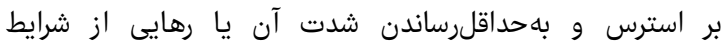

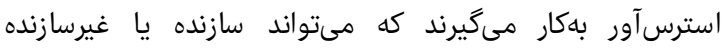

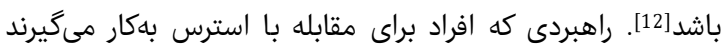

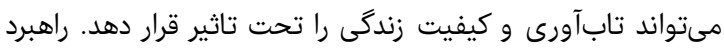

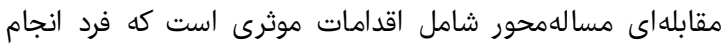

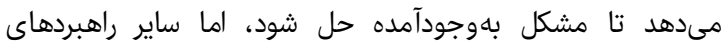

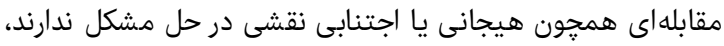

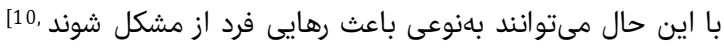

آشفتگى دهاى هيجانى و مشكلات روانشناختى ناشى از آسيبهاى

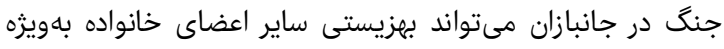

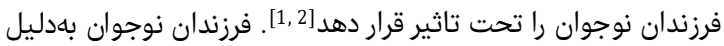

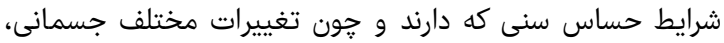

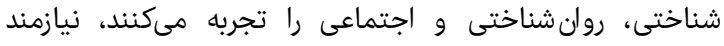

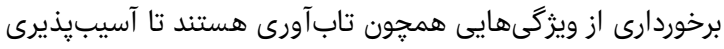

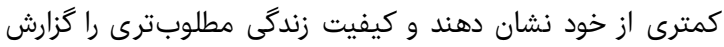

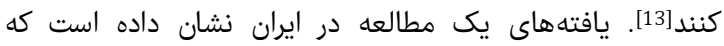

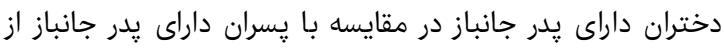

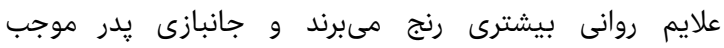

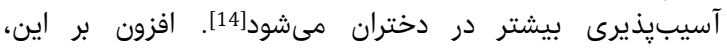

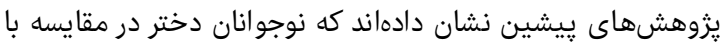

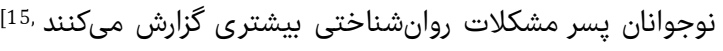

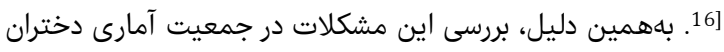

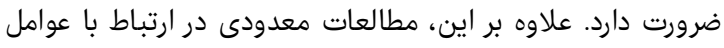

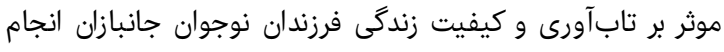

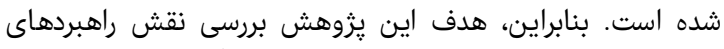

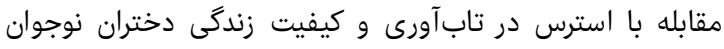

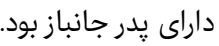

\section{نقش راهبردهاى مقابله با استرس در تابآورى و و كيفيت زندكى دختران نوجوان داراى يدر جانبان}

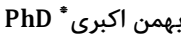

كروه روانشناسى عمومى، دانشكده علوم انسانى، واحد رشت، دانشكاه آزاد

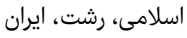

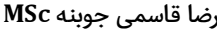

كروه مشاوره، دانشكده روان شناسى و علوم تربيتى، دانشكاه خوارزمى، تهران،

ايران

MSc معصومه اسدى يرور

كروه روانشناسى عمومىى، دانشكده علوم انسانى، واحد رشت، دانشگاه آزاد

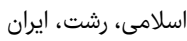

קكيده

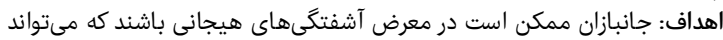

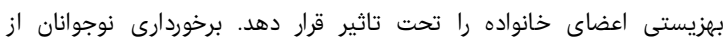

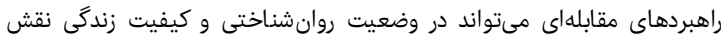

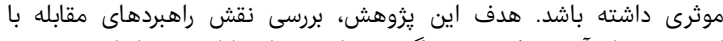

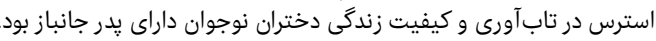

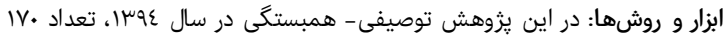

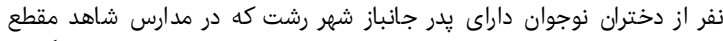

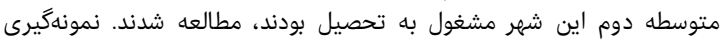

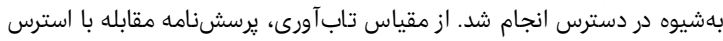

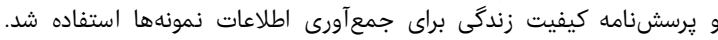

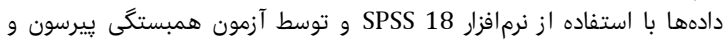

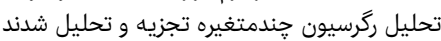

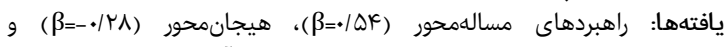

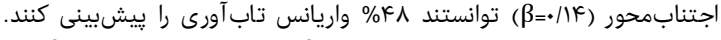

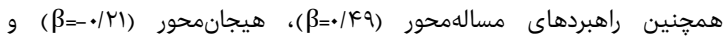

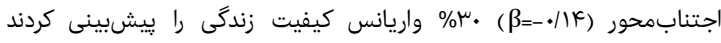

. $(\mathrm{p}<\cdot / \cdot 1)$

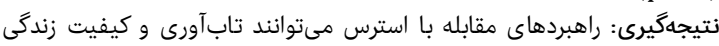

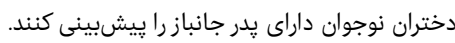
كليدوازٔها: راهبردهاى مقابله، تاب آورى روانى، كيفيت زندكىى، نوجوان

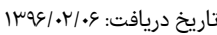

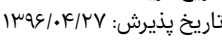

bakbari44@yahoo.com :نويسنده مسئول:

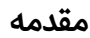

جانبازان بهواسطه شرايط خاص، ممكن است در معرض برخى إنى

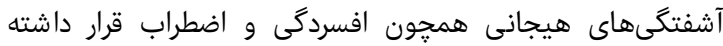

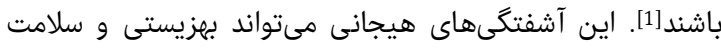

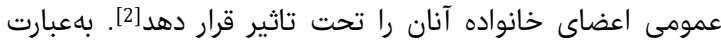

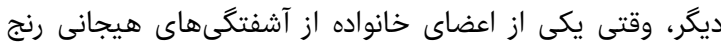

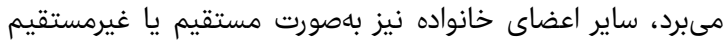

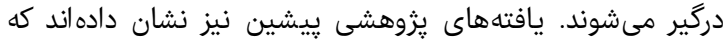

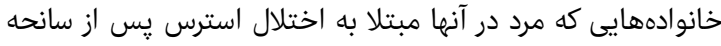

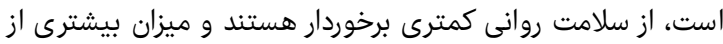

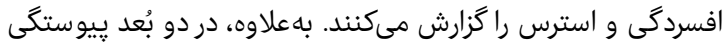

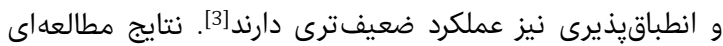

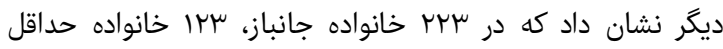

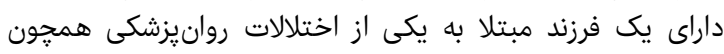

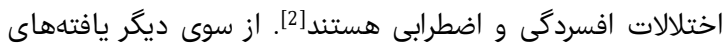

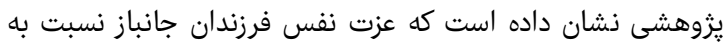

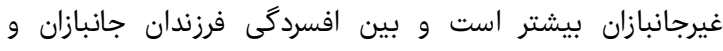

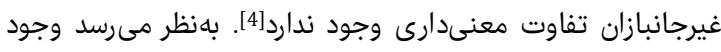




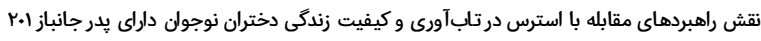

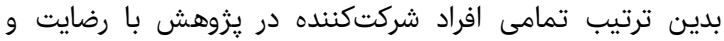

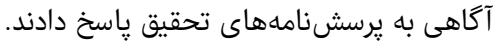

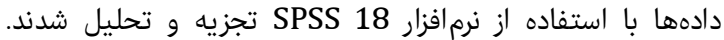

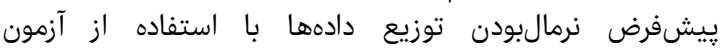

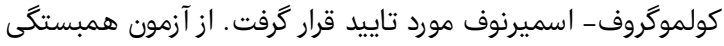

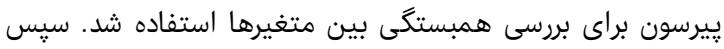

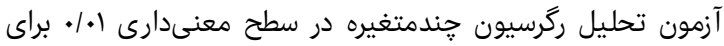

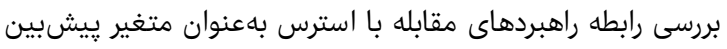

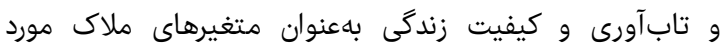

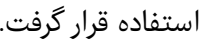

ميانگين نمره تابهآ ميآورى با ميانگين نمرات سبك مقابلهای

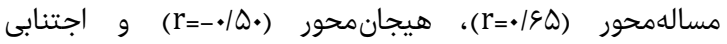

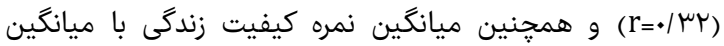

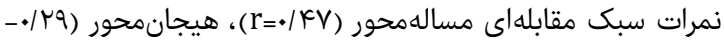

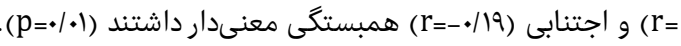

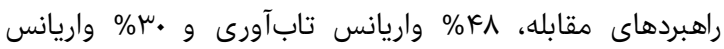

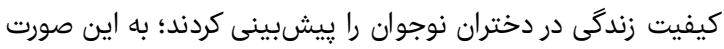

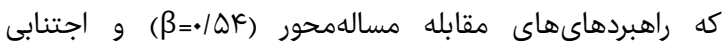

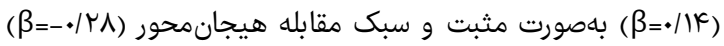

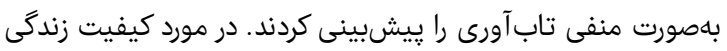

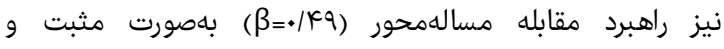

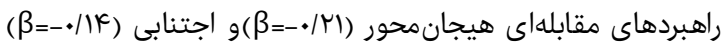

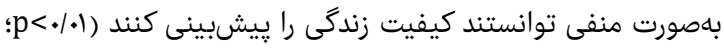

جدول () ميانكين آمارى نمرات تابآورى، كيفيت زندكى و راهبردهاى مقابله با دإن

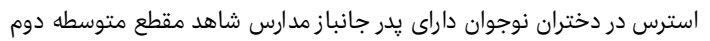

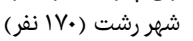

\begin{tabular}{|c|c|}
\hline نمرات & متغيرها \\
\hline$\Delta r / \wedge ৭ \pm 9 / \Delta$. & سبك مسالهمحور \\
\hline$\kappa \varepsilon / 9 \wedge \pm 9 / 1$. & سبك هيجان محور \\
\hline KN/KS $\pm N / T$. & سبك اجتنابمحور \\
\hline$\wedge \mu / \wedge \Delta \pm 1 \cdot / \wedge q$ & كيفيت زندگى \\
\hline $9 \cdot / \mu \cdot \pm 10 / 1 T$ & تابآورى \\
\hline
\end{tabular}

در اين يزوهش، نقش راهبردهاى مقابله با استرس در تابآورى و دخد

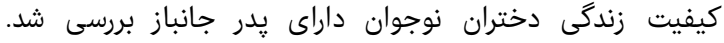

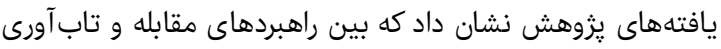

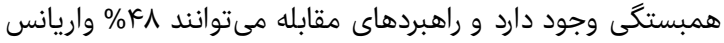

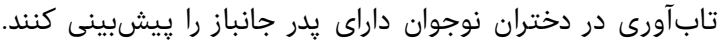

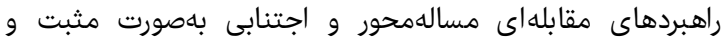

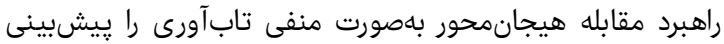
كردند. يافتههاى ساير مطالعات با اين يزوهش همور همخوانى دارد-20]

يافتههاى يك بررسى همسو با ڤيزوهش حاضر نشان داد كه

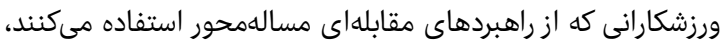

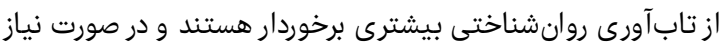

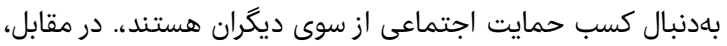

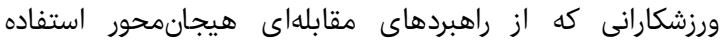

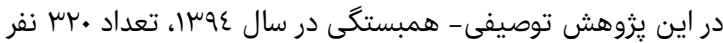

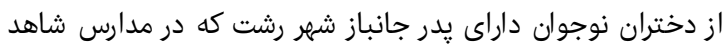

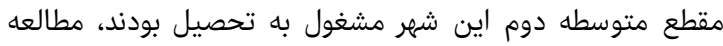

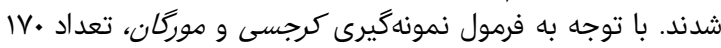

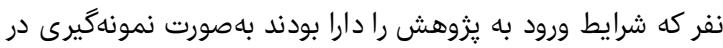

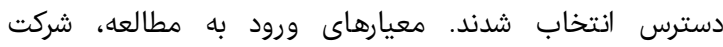

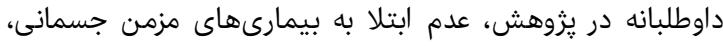

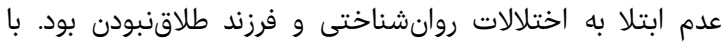

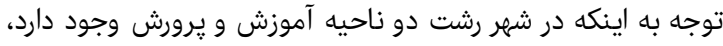

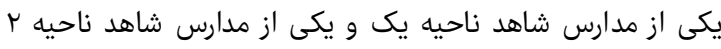

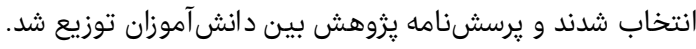

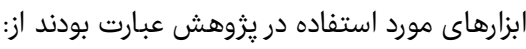

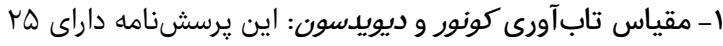

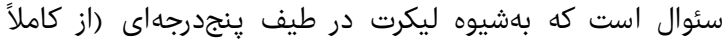

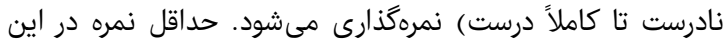

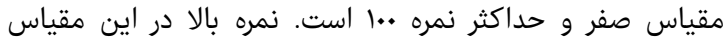

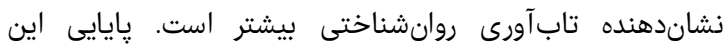

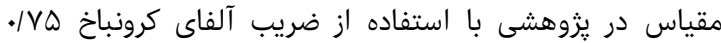

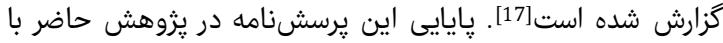

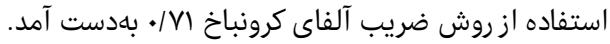

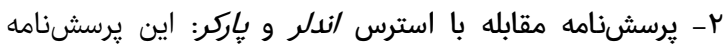

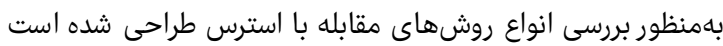

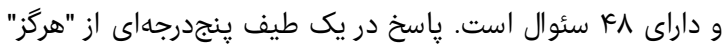

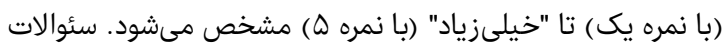

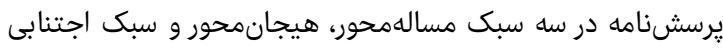

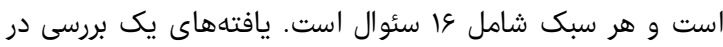

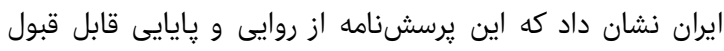

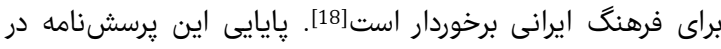

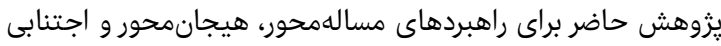

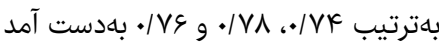

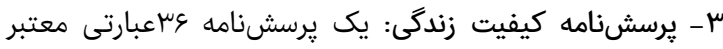

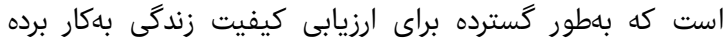

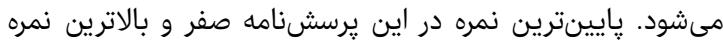

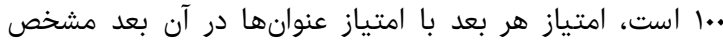

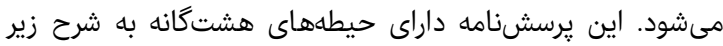

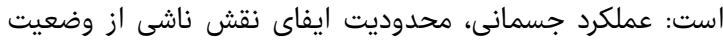

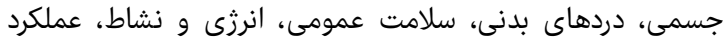

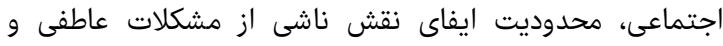

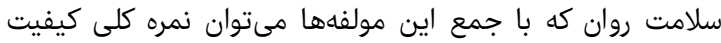

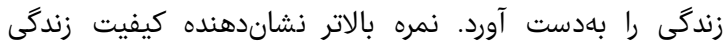

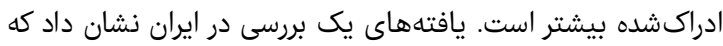

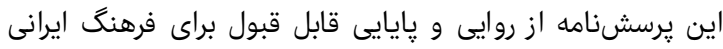

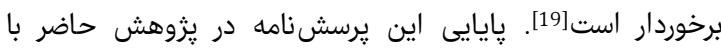

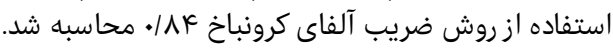

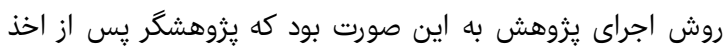

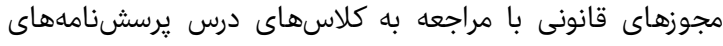

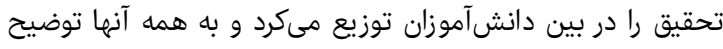

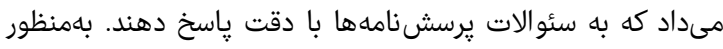

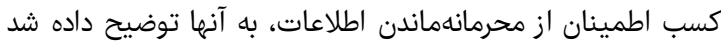

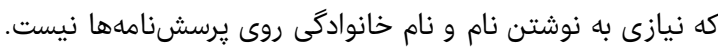




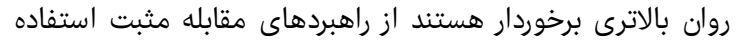

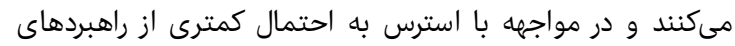

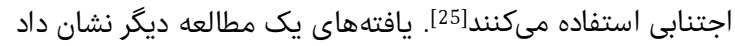

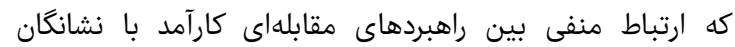

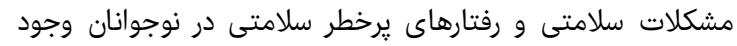

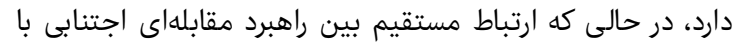
نشانكان مشكلات سلامتى و رفتارهاى يرخطر سلامتى وجود إنى داردمان]

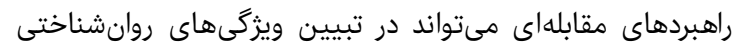

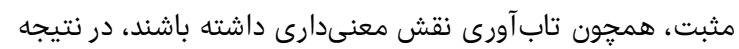

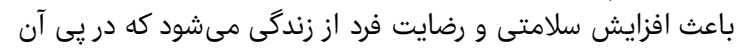

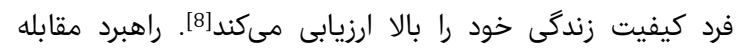

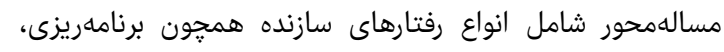

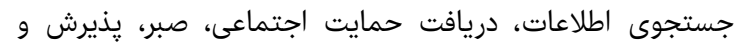

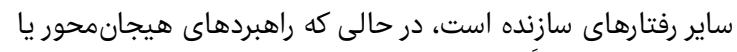

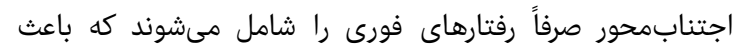

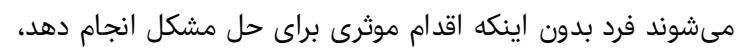

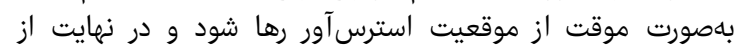

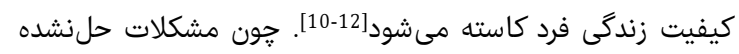

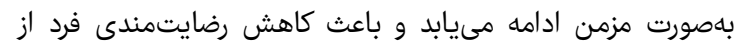

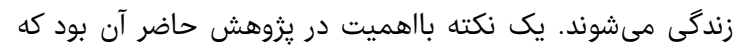

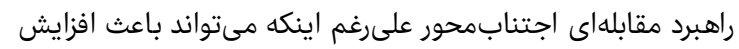

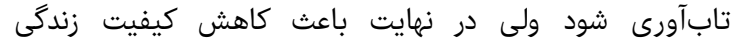
ادراكشده در فرد مى شود.

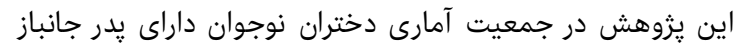

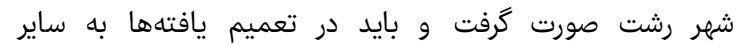
جمعيتهاى آمارى احتياط كرد.

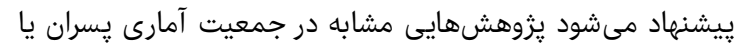

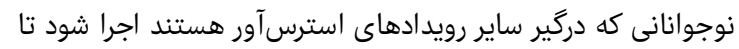

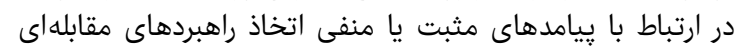
مختلف شناخت بيشترى حاصل شود.

نتيجه رهيرى

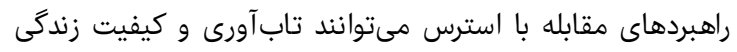

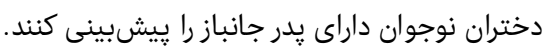

تشكر و قدردانى: از دانشآموزان محترم كه در يزوهش حاضر شركت

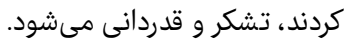

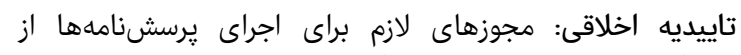

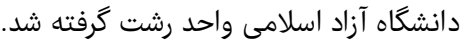

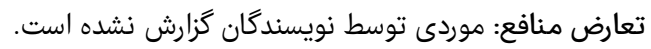

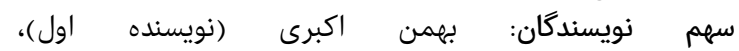

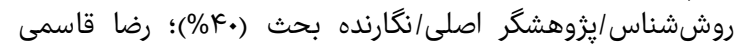

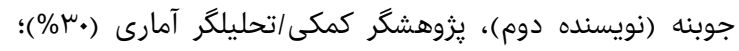

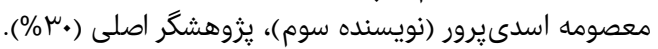

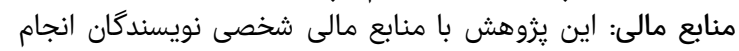

منابع

1- Eskandari H. The survey of veterans' depression and anxiety and its relationship with veterans' socialeconomic activities. J Mil Med. 2015;16(4):197-203. [Persian] ميكىنند تابآورى كمتر نشان مىدهند و و تمايل به سرزنشكركردن

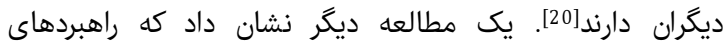

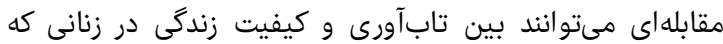

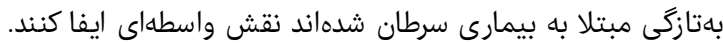

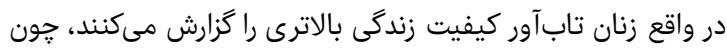

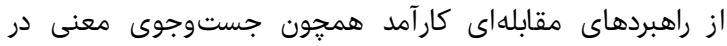

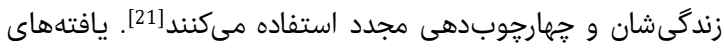

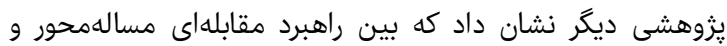

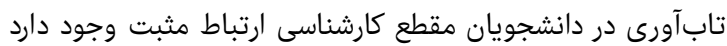

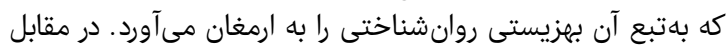

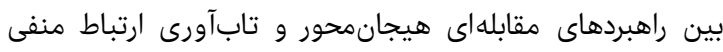

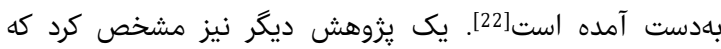

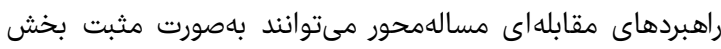
قابل توجهى از واريانس تابآورى در بيماران قلبى را تبيين كند[23]. تون.

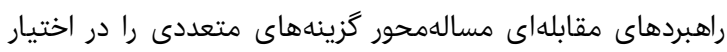

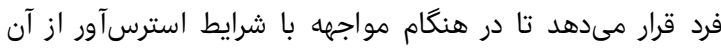

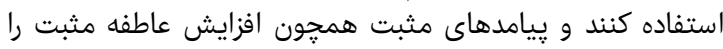

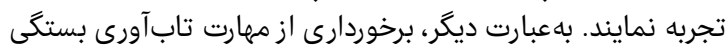

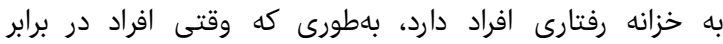

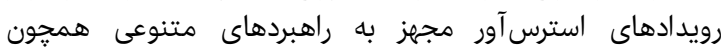

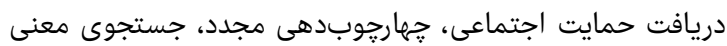

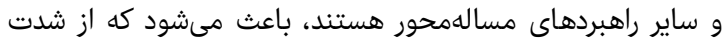

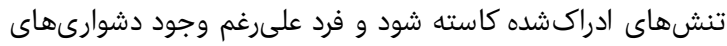

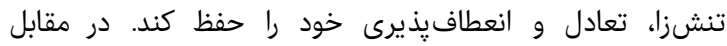

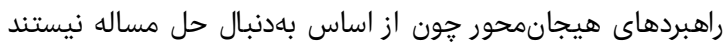

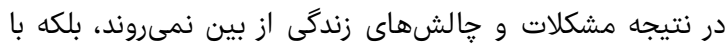

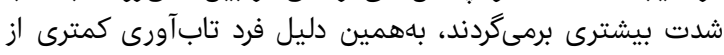

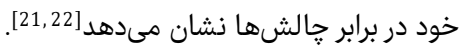

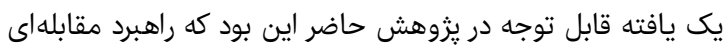

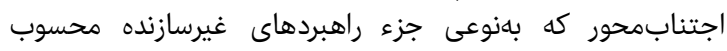

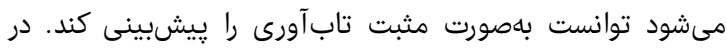

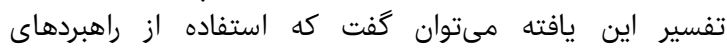

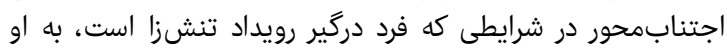

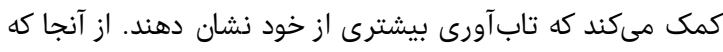

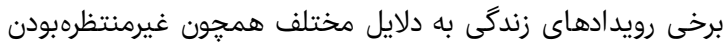

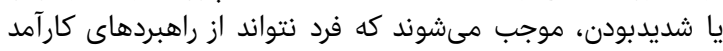

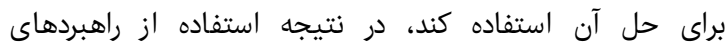

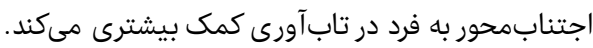

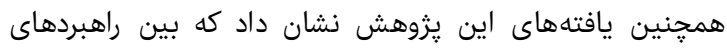

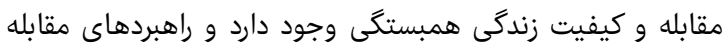

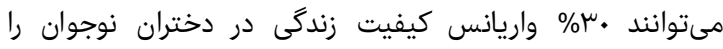

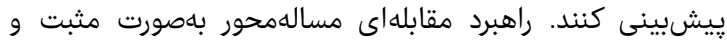

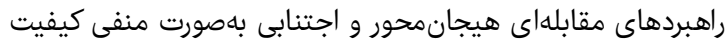

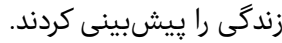
يافتهاى يكى براى برسى نشان دان داد كه بين راهبردهاى مقابله

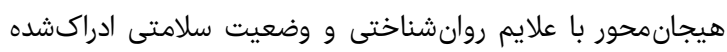

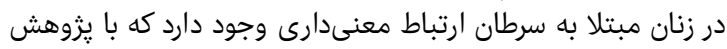

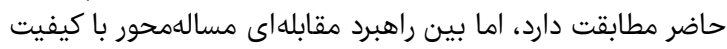

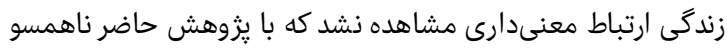

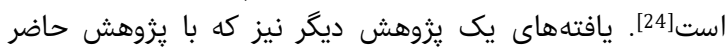

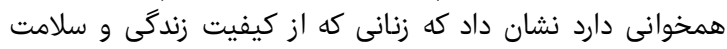




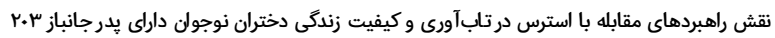
15- Roshan Chasaly R, Sharbati A, Rahimi Z. Comparison of mental health of high school students according to gender and place of residence. J Tran Learn Res. 2011;1(45):71-8. [Persian]

16- Zamani F, Shahri P, Zareei E. Mental health status of high school girl and boy students in boarding schools in Markazi Province. Jundishapur J Health Sci. 2012;4(1):11-9. [Persian]

17- Zarifi M, Honari H, BahrolOloum H. Psychometrics properties and normalization of persi version of resiliency questionnaire among athletes and nonathletes students. Sport Psychol Stud. 2016;5(15):13150. [Persian]

18- Ghoreyshi Rad F. Validation of Endler \& Parker coping scale of stressful situations. J Behav Sci. 2010;4(1):1-7. [Persian]

19- Montazeri A, Goshtasebi A, Vahdaninia M, Gandek B. The short form health survey (SF-36): Translation and validation study of the Iranian version. Qual Life Res. 2005;14(3):875-82.

20- Yi JP, Smith RE, Vitaliano PP. Stress-resilience, illness, and coping: A person-focused investigation of young women athletes. J Behav Med. 2005;28(3):257-65.

21- Manne SL, Myers-Virtue S, Kashy D, Ozga M, Kissane D, Heckman C, et al. Resilience, positive coping, and quality of life among women newly diagnosed with gynecological cancers. Cancer Nurs. 2015;38(5):375-82. 22- Smith MM, Saklofske DH, Keefer KV, Tremblay PF. Coping strategies and psychological outcomes: The moderating effects of personal resiliency. J Psychol. 2016;150(3):318-32.

23- Lee S, Kim S, Young Choi J. Coping and resilience of adolescents with congenital heart disease. J Cardiovasc Nurs. 2014;29(4):340-6.

24- Khalili N, Farajzadegan Z, Mokarian F, Bahrami F. Coping strategies, quality of life and pain in women with breast cancer. Iran J Nurs Midwifery Res. 2013;18(2):105-11.

25- Mirabzadeh A, Baradaran Eftekhari M, Setareh Forouzan A, Sajadi H, Rafiee H. Relationship between ways of coping and quality of life in married women: Toward mental health promotion. Iran Red Crescent Med J. 2013;15(8): 743-8.

26- Steiner H, Erickson SJ, Hernandez NL, Pavelski R. Coping styles as correlates of health in high school students. J Adolesc Health. 2002;30(5):326-35.
2- Mozafari M, Ghanizadeh A, Ashkani H, Firoozabadi A, Alishahi MJ, Dehbozorgi GH, et al. The prevalence of anxiety and depression disorders in the children of veterans of Shiraz, Iran: A case control study. J Qom Univ Med Sci. 2009;3(2):19-24. [Persian]

3- Mohseni M, Farnia MR, Taghva A, Dehghan Manshadi Z, Rezaei Fard A. Effect of having a post-traumatic stress disordered man on the quality of life, depression, stress, anxiety and structure of the family. Iran J War Public Health. 2014;6(5):207-14. [Persian]

4- Rahmani N, Akbar Natayej K, Alipoor H. Assessment and comparison of self esteem and depression in war handicappeds and non war handicappeds children of shahed guidance schools in Sari City. Iran J War Public Health. 2012;4(4):29-34. [Persian]

5- Southwick SM, Bonanno G, Masten A, Panter-Brick C, Yehuda R. Resilience definitions, theory, and challenges: Interdisciplinary perspectives. Eur J Psychotraumatol. 2014;5:1-14.

6- Stewart DE, Yuen T. A systematic review of resilience in the physically ill. Psychosomatics. 2011;52(3):199209.

7- Wu G, Feder A, Cohen H, Kim JJ, Calderon S, Charney DS, et al. Understanding resilience. Front Behav Neurosci. 2013;7:10.

8- Nawaz A, Malik JA, Batool A. Relationship between resilience and quality of life in diabetics. J Coll Physicians Surg Pak. 2014;24(9):670-5.

9- Khanna D, Tsevat J. Health-related quality of life--an introduction. Am J Manag Care. 2007;13(9):218-23.

10- Hampel P, Petermann F. Perceived stress, coping, and adjustment in adolescents. J Adolesc Health. 2006;38(4):409-15.

11- Frydenberg E, Lewis R. Relations among well-being, avoidant coping, and active coping in a large sample of Australian adolescents. Psychol Rep. 2009;104(3):74558.

12- Carver CS, Connor-Smith J. Personality and coping. Annu Rev Psychol. 2010;61:679-704.

13- ElenaAnghel R. Psychological and educational resilience in high vs. low-risk Romanian adolescents. Procedia Soc Behav Sci. 2015;203:153-7.

14- Radfar S, Haghani $H$, Tavalaei SA, Modirian E, Falahati M. Evaluation of Mental health state in veterans family (15-18 Y/O adolescents). J Mil Med. 2005;7(3):203-9. [Persian] 